\title{
IMPLEMENTATION OF CONTACT DEFINITIONS CALCULATED BY FEA TO DESCRIBE THE HEALING PROCESS OF BASAL IMPLANTS
}

\author{
Stefan Ihde ${ }^{\mathrm{a} *}$, Tomas Goldmann ${ }^{\mathrm{b}}$, Lucie Himmlova ${ }^{\mathrm{c}}$, Zoran Aleksic ${ }^{\mathrm{d}}$, Jiri Kuzelka ${ }^{\mathrm{b}}$
}

\author{
${ }^{a}$ Gommiswald Dental Clinic, Dorfplatz 11, 8737 Gommiswald/SG, Switzerland \\ ${ }^{b}$ Czech Technical University in Prague, Laboratory of Biomechanics, Department of Mechanics, Biomechanics and \\ Mechatronics, Faculty of Mechanical Engineering, Technicka 4, 16667 Prague, Czech Republic \\ c Institute of Dental Research ${ }^{{ }^{s t}}$ Medical Faculty of Charles University and General Medical Hospital, Vinohradska 48, \\ 12021 Prague 2, Czech Republic \\ ${ }^{a}$ Belgrade School of Dentistry, Department of Periodontology, Dr. Subotic str. 4, 11000 Belgrade, Serbia \\ e-mail:drihde@bluewin.ch
}

Received: March 10, 2008; Accepted: April 3, 2008

Key words: Progress of implant osseointegration/Finite element analysis (FEA)/Implant/Bone interaction \& stresses/Implant fatigue testing methods/Intrinsic gradual stress shifting

\begin{abstract}
Aims: Bone structure around basal implants shows a dual healing mode: direct contact areas manifest primary osteonal remodeling, in the void osteotomy-induced spaces, the repair begins with woven bone formation. This woven bone is later converted into osteonal bone. The purpose of this study was to develop a model to accurately represent the interface between bone and basal implant throughout the healing process. The model was applied to the biological scenario of changing load distribution in a basal implant system over time.

Methods: Computations were made through finite element analysis using multiple models with changing boneimplant contact definitions which reflected the dynamic nature of the interface throughout the bony healing process. Five stages of bony healing were calculated taking into account the changes in mineral content of bone in the vicinity of the load transmitting implant surfaces.

Results: As the bony integration of basal implants proceeds during healing, peak stresses within the metal structure shift geographically. While bony repair may still weaken osteonal bone, woven bone has already matured. This leads to changes in the load distribution between and within the direct contact areas, and bone areas which make later contact with implant.

Conclusions: This study shows that basal implants undergo an intrinsic shift of maximum stress regions during osseointegration. Fatigue testing methods in the case of basal implants must therefore take into account this gradual shift from early healing phase until full osseointegration is achieved.
\end{abstract}

\section{INTRODUCTION}

Injured bone tends to soften, not only in the region of trauma itself but also in a large surrounding area which may be indirectly injured or not injured at all. This is owing to the fact that the remodeling process extends from non-injured areas through repair areas and deep into the native, old bone ${ }^{1}$. Secondary osteons, the actual morphological units of remodeling (BMUs) may reach up to $20 \mathrm{~cm}$ long $^{2}$. Like a drilling head they move forward at a speed of $40 \mu \mathrm{m}$ per day while full remineralization of the osteonal zones after any injury or surgical trauma may take up to two years ${ }^{3}$.

Bone structure around basal implants shows a dual mode of healing: Whereas direct contact areas show primary osteonal remodelling, in the void osteotomy-induced spaces, the repair starts with woven bone formation. This woven bone is later converted into osteonal bone $e^{4}$.

Use of "SOFT" and "HARD" as contact definitions $s^{5,6}$ to characterize and/or categorize bone rigidity in interaction with the basal implant surface has already been described $^{6}$. Both contact definitions were applied to the interaction between the implant shaft and the bone (with the shaft providing a simple geometry). In this article the same definitions are applied to a real bone situation in a human mandible.

The preliminary study gave us the opportunity to use either contact definition for describing the process of the bony healing and the integration process of the implants. Since softening and remineralisation are normal events in all bone healing, implant stability will change over time. For basal implants we calculated five steps of bone healing to enable us to describe the process of osseointegration in different areas of the basal implants, as well as at different timelines postoperatively.

\section{MATERIAL AND METHODS}

The geometry of the mandible was gained from CTscans of a real edentulous mandible. The finite element mesh of the mandible was created in the system ABAQUS 6.6-5 (Abaqus Inc., Providence, RI 02909-2499, USA) by the C3D4 element type. The material model used de- 
fined bone for this study as an inhomogenic, linear elastic isotrophic material. Inhomogenic material properties were obtained from the greyscale values of $\mathrm{CT} /$ scans of this mandible, using the areas of the second molar for the single baseplate implant, and the area of the canine for the triple-baseplate implant. The greyscale values were transformed to a 100 linear elastic material model (Young modulus (E) and Poisson Ration $(\mu))$. The grayscale was calibrated, using point 14 (Fig. 1) of the scaling measured by Schwartz-Dabney \& Dechow ${ }^{7}$. Adequate insertion slots were prepared to enable the insertion of two different basal implants into the mandibular model. The implants were differently designed and the whole system, consisting of four implants and a fixed bridge was loaded as proposed by Mericse-Stern ${ }^{8}$ (114.6 N vertical, $17.1 \mathrm{~N}$ vestibular/oral, $23.4 \mathrm{~N}$ horizontal forces) the latter being present between implants. Defined forces were exerted separately on each implant, assuming that the implants were not splinted. The boundary conditions were visualized and explained in Fig. 2.

The material of implants is assumed to be linear elastic. The single baseplate implant (Fig. 3) is threaded into the upper shaft area. This thread is designed for bolting to abutment, which is also part of this model (Fig. 2). The triple baseplate implant (Fig. 4) is made as a single piece (the abutment is part of the implant). Implants are made from Ti Grade $1\left(\mathrm{E}=1.0 \times 10^{5} \mathrm{MPa}, \mu=0.340\right)$ and the abutment with thread is manufactured from Ti6Al4V $(\mathrm{E}=113860 \mathrm{MPa}, \mu=0.342)$.

Basal implants are primarily anchored at the baseplates within the cortical areas of the bone. In order to describe the healing process we defined different contact surfaces on each implant taking into account the development of different contact properties over time. The contact properties ranged from "HARD" to "normal SOFT" 3,6 .

The following three areas are generally distinguished:

1. the area of cortical engagement of the baseplate(s),

2. the endosseous area which we assumed was immediately postoperatively filled with blood or replaced by non-load-transmitting spongious bone which had no contact with implant, and

3 . the area of crestal bone penetration where late cortical osseointegration is typically observed (Fig. 3, 4, 6).

Based on these assumptions and conditions, the following calculations were done

1. At baseline no contact between bone and the implant is considered to be present in areas 2 and 3 . The implant is in contact with cortical bone in area 1 (except for the crestal slot gap stemming from the insertion of the vertical implant part which was not taken into consideration in our calculation).

2. From histological results, we postulated a healing situation where the baseplate is anchored in cortical but remodelled bone (which we considered SOFT), while the upper vertical implant part is integrated with newly formed osteonal or woven bone as well. The contact was also defined as SOFT (Fig. 5). For the calculation no contact was assumed to be present in area 2.

3. This situation represents a later phase of healing where the cortical areas have been completely remineralized and therefore this contact (area 1, Figs. 3, 4) was considered HARD. The crestal penetration area as well as the spongious area (areas 3 and 2, Figs. 3, 4) were assumed to be still under remodeling and were set as "SOFT".

4. At an even more advanced phase of healing, all cortical areas (1 and 3) were considered "HARD", while the area of the spongious bone (2) was still assumed to be "SOFT".

5. Long lasting integration of an implant body inside the bone will lead to corticalization of the entire endosseous surfaces. This situation was simulated and calculated by defining all contact definitions as "HARD".

\section{RESULTS AND DISCUSSION}

The results are displayed in Fig. 5. The stress distribution was computed in von Mises stress scale and identified for each implant type as well as for the bone areas around each implant. (Note that although Fig. 5 shows an identical colour, the scales for the side of the bone and the implant differ.) We had to set the scaling differently in order to make stress graduations visible. For bone, a maximum stress of $105 \mathrm{MPa}$ was chosen and for Titanium (Grade 2) a maximum of $400 \mathrm{MPa}$ was selected. Grey areas are above these limits. Note that the stress peak just under the thread of the single baseplate implant is highest and is caused by the notch. This peak does not occur in the design of the triple baseplate implant. This result clearly advocates the use of the one-piece implant (Fig. 4) instead of threaded implant (Fig. 3) in a view of stress distribution.

Our results are valid for a hollow bone with spongious filling but cannot be applied for non-augmented transsinusal placements of basal implants where no contact is expected in area 2.

During healing of bony wounds, the osteonal remodelling resorbs highly mineralized areas of bone and replaces it by bone matrix. The remaining (i.e. not remodelled) mineralized areas of old bone have to carry a greater load than the baseline before the start of this process. If parts of these areas should fail through overload osteolysis, micro-cracks inside the bone accumulate leading to a total structural failure, a state which is described as overload osteoporosis. For this reason, living bone fails significantly earlier than dead bone, the latter being able to cope with up to 1-3 millions of load cycles until it changes into a flexible composite laminate material. Basal implants may be installed in minimal rests of native bone; however one must take care, not to overload either bone or the implants themselves. One strategy to avoid overloading is to reduce the masticatory forces through medication ${ }^{3}$.

Clinically it can be observed, that basal implants, which are prosthetically overloaded in an early phase of bone healing, usually become mobile inside the bone, 

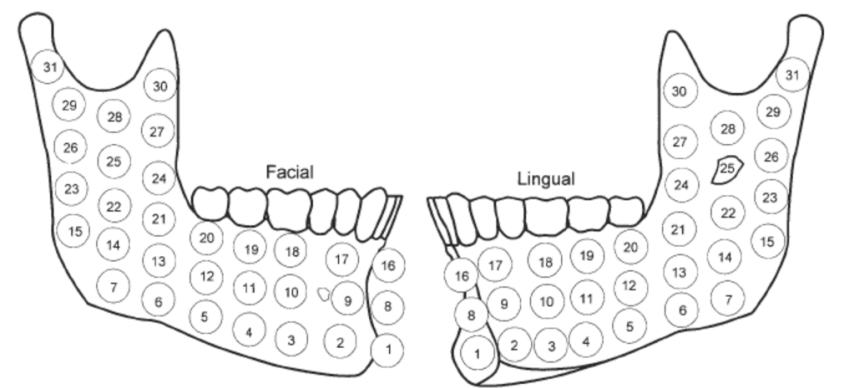

Fig. 1. Calibration of material model of the mandible according Schwartz-Dabney? ${ }^{7}$ Greyscales of our model were calibrated at point 14 , facial side.

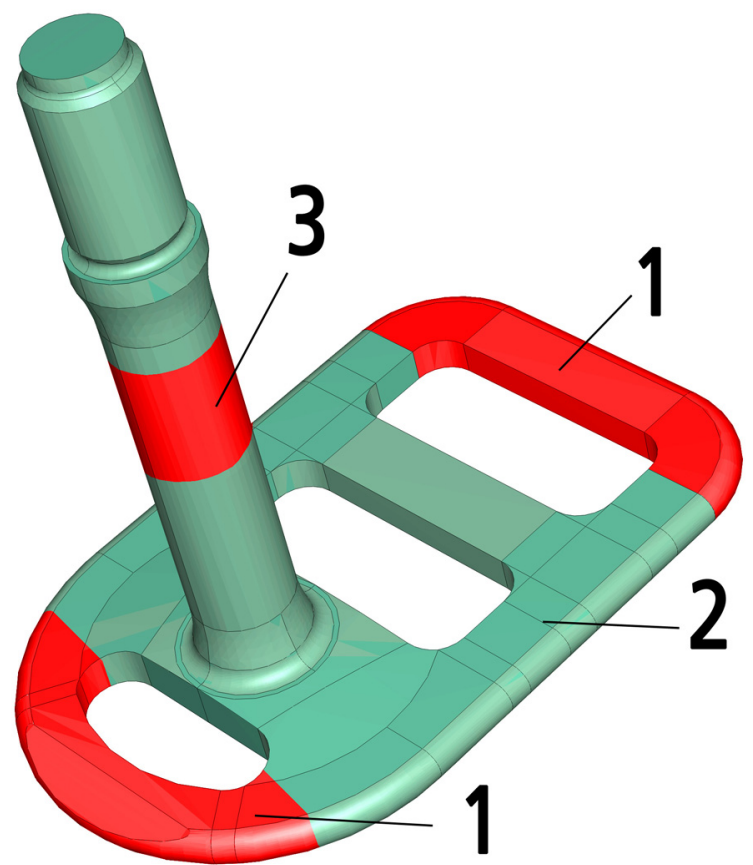

Fig. 3. Single baseplate implant with basal cortical anchorage regions (1), a crestal trans-cortical region (3), and a spongious region (2).

but they never fracture. Only well-integrated implants can fracture: they either break at the baseplate (between 12 - 36 month after placement; Fig. 5 Stage 1) or in the neck area (later than 36 months after placement, Fig. 5 Stage 5). The results of our calculations (Fig. 5) match exactly these clinical finding.

Our model does not consider a plastic model of titanium implant. Only the elastic behaviour has been taken into account. We presuppose that plastic deformation will not occur during early stages of the healing process, as the

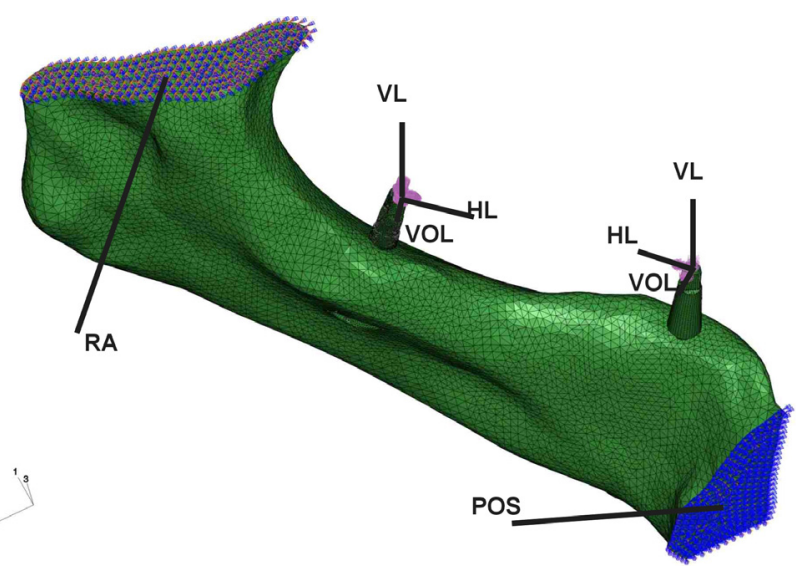

Fig. 2. Boundary conditions of the mandible. $\mathrm{POS}=$ plane of symmetry of the entire model; RA = ramus ascendens, fixed plane; $\mathrm{VL}=$ vertical load $114,6 \mathrm{~N} ; \mathrm{HL}=$ horizontal load $17.1 \mathrm{~N} ; \mathrm{VOL}=$ vestibulo-oral load 23,4 N.

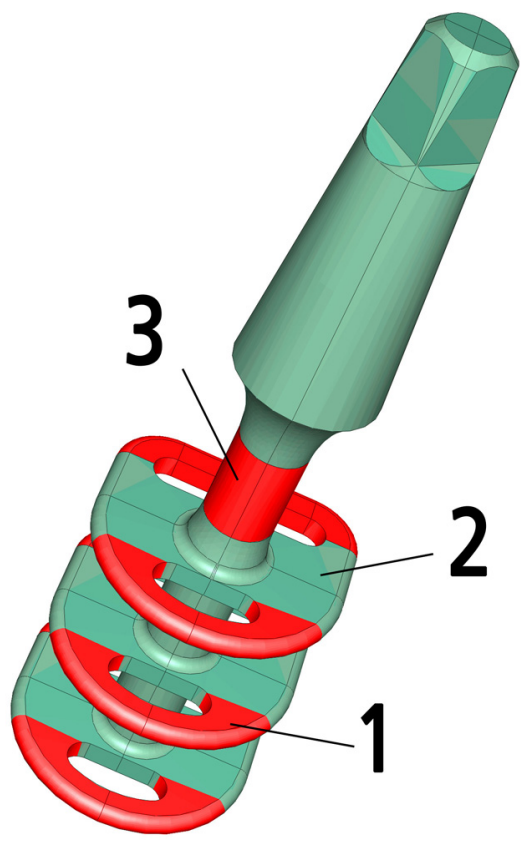

Fig. 4. Triple baseplate implant with six basal cortical anchorage regions (1), a crestal, trans-cortical region, and a spongious region. Out of the six cortical anchorage regions, at least one on each side should have cortical contact.

bone is too soft to provide sufficient resistance and would tend to fail through micro-crack accumulation.

One of our assumptions is that the implantation sites remain in the same relative position to each other. It should not be forgotten that this stability is not guaranteed and that the jaw bones may change their relative length and height in relation to the site of the basal implant $^{9}$ and that osseointegrated implants are less able to compensate for such changes (e.g. through orthodontic movement) than teeth. If the relative position of the base 


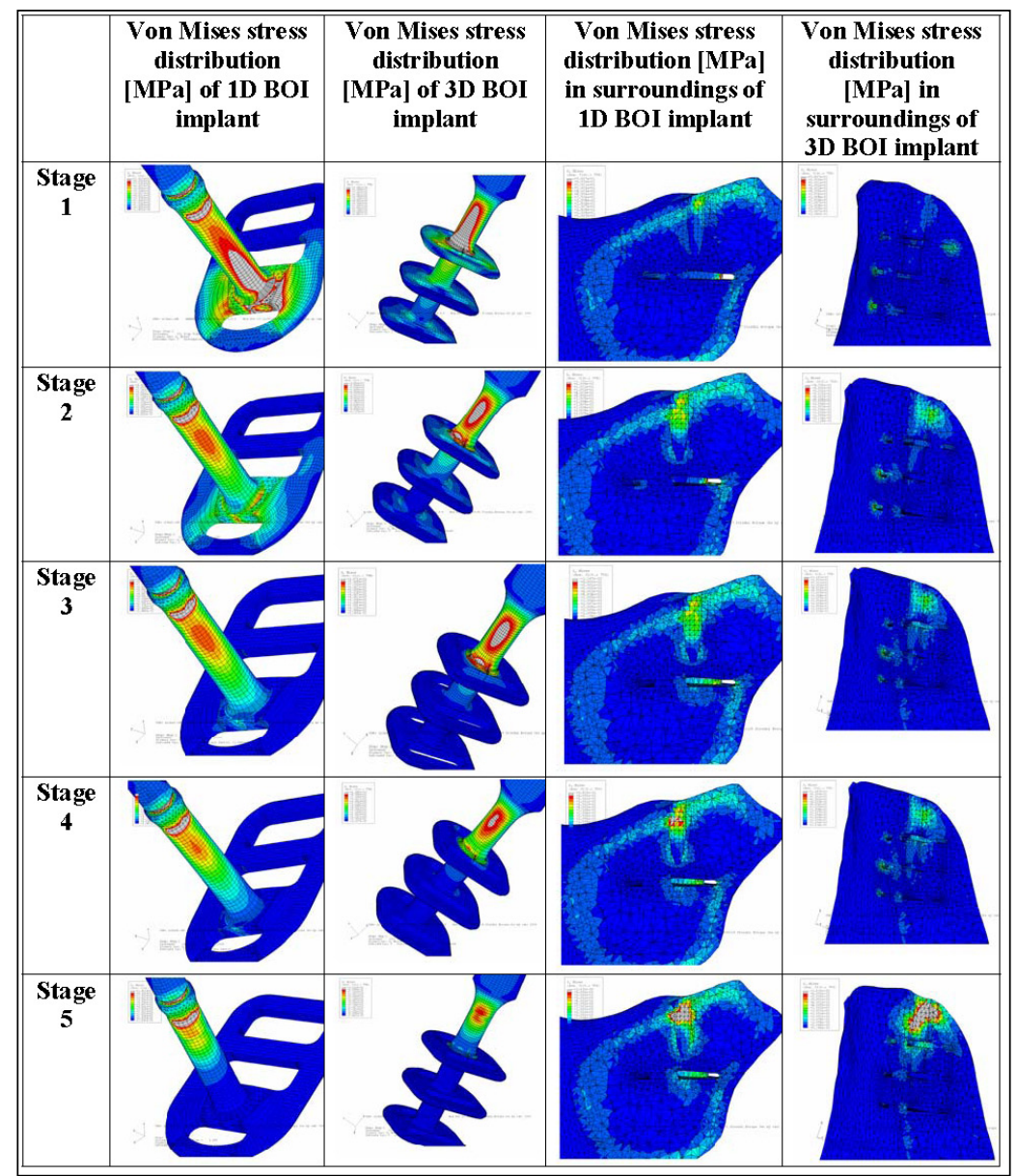

Fig. 5. Visualization of the results through adjusted color scales for the bone and the implant side of the contact.

Table 1. Overview on contact definitions at different stages of healing in the areas defined in Fig. 4 and Fig. 5.

\begin{tabular}{|c|c|c|c|}
\hline & Area1 & Area 2 & Area 3 \\
\hline Stage 1 & SOFT & missing & missing \\
\hline Stage 2 & SOFT & missing & SOFT \\
\hline Stage 3 & HARD & SOFT & SOFT \\
\hline Stage 4 & HARD & SOFT & HARD \\
\hline Stage 5 & HARD & HARD & HARD \\
\hline
\end{tabular}

plates changes, a pre-tension may develop within the implant body. As a result, even lower cyclic forces may lead to a situation where the elastic range of deformation is exceeded, leading to an earlier failure of the affected implants. When creating fixed bridges on teeth and basal implants, the effect may be even greater, since teeth can perform orthodontic movements. This should be taken into account when designing basal implants as well as implant-prosthetical systems.

Basal implants do not show the downward path called "peri-implantitis", which has been described for screw designs, because their surface is completely machined and the diameter of the vertical penetration area is thin. Vertical, crater-like bone loss is not observed in the designs discussed here ${ }^{3}$. While in axial implants the maximum stress zones move towards apical direction and while the bone loss is rather delayed, the opposite development is seen in basal implants: their maximum stress zones move in the crestal direction, while the process of integration progresses.

The results of our study suggest that in softer graduations of Titanium, the elastic range of deformation may be exceeded and that the design of the implants should provide structurally reinforced regions.

\section{CONCLUSIONS}

During the healing of bone around dental basal implants the area of the load transmission changes. Since 


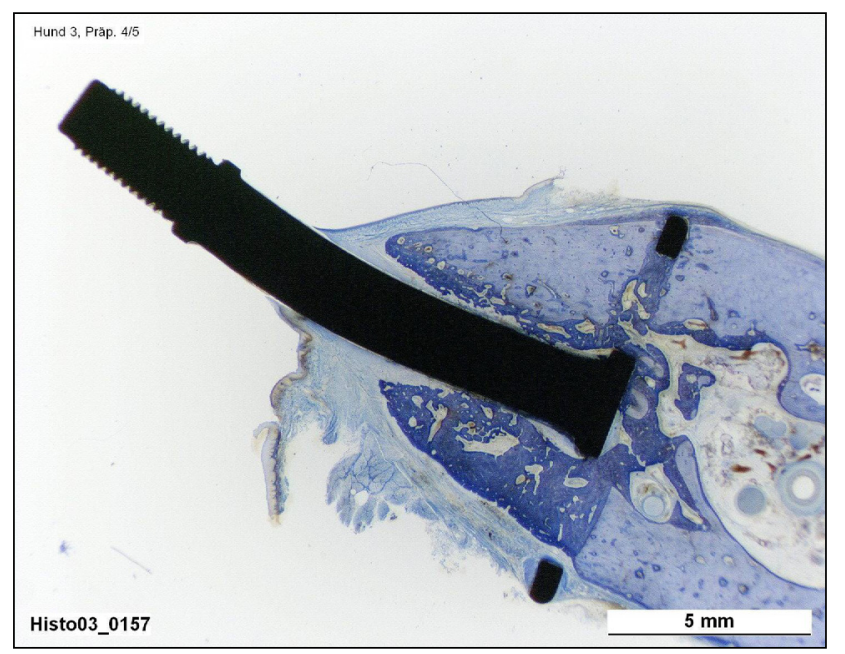

Fig. 6. Transsection through an immediately loaded basal implant, 3.5 month postoperatively. Note that the baseplate is cut vertically. New bone areas are dark blue, old bone light blue. The integration progresses from several directions through gap jumping and osseoadaptation. (From: Ihde S.: Principles of BOI; with permission of Springer Publ. Heidelberg.)

the vertical implant areas participate little in the load transmission after implant placement, a considerable part of the load is transmitted via the base plates during this period (Fig. 5 Stage 1). In a later phase of healing and after the reconstitution of bone, a larger part of the non-axial forces are transmitted through the collar area of basal implants (area 3, Figs. 3, 4; and Fig. 5 Stage 5) into the crestal bone segments.

Basal implants, owing to their design and the dual healing process induced by their insertion, have two different regions of maximum stress. However, the position of the most critical stress region mainly depends on the time which has passed after the insertion.
Tests for fatigue resistance will have to take this gradual shift of maximum stress regions into account. Standard testing procedures according to the standard EN ISO 14801:2006 (ref. ${ }^{10}$ ) may not be directly applicable and have to be adapted to this implant design. Experiences from long term usage of basal implants should be considered as a realistic reference.

\section{ACKNOWLEDGEMENTS}

This study was funded by the Czech Ministry of Education project No. MSM 6840770012 and by the Grant Agency of the Czech Republic under project No. 106/06/0849

\section{REFERENCES}

1. Atkinson PJ, Powell K, Woodhead C. Cortical structure of the pig mandible after the insertion of metallic implants into alveolar bone. Arch Oral Biol 1977; 22:383-91.

2. Burr M. Skeletal tissue mechanics. New York: Springer; 1998 , ISBN.

3. Ihde S. Clinical note. CMF Impl. Dir. 2006 (1).

4. Ihde S. Principles of BOI. Heidelberg: Springer; 2005, ISBN 3-54021665.

5. Abaqus manual: www.abaqus.com

6. Ihde S, Goldmann T, Himmlova L, Alexic Z. Use of finite element analysis to model bone-implant contact with basal implants. Oral Surg Oral Med Oral Pathol Oral Radiol Endod., accepted - in press 2008.

7. Schwartz-Dabney CL, Dechow PC. Variations in cortical material properties throughout the human dentate mandible. Am J Phy Anthropol 2003; 120:252-277.

8. Mericske-Stern R, Assal P, Buergin W. Simultaneous force measurements in 3 dimensions on oral endosseous implants in vitro and in vivo: A methodological study. Clin Oral Impl Res 1996; 7:378386.

9. Ihde S. Adaptations fonctionnelles de la hauteur de l'os péri implantaire aprčs implantation de BOL Implantodontie - Implants et dentisterie esthétique 2003; 12(4):23-33.

10. EN ISO 14801:2006 - Dentistry - Implants - Dynamic fatigue test for endosseous dental implants (ISO/DIS 14801:2006) 